\title{
Reproducibility and Experimental Design for Machine Learning on Audio and Multimedia Data
}

\author{
Gerald Friedland \\ fractor@eecs.berkeley.edu \\ University of California, Berkeley \\ Berkeley, CA
}

\begin{abstract}
This tutorial provides an actionable perspective on the experimental design for machine learning experiments on multimedia data. The tutorial consists of lectures and hands-on exercises. The lectures provide an engineering introduction to machine learning design. By understanding the information flow and quantities in the scientific process, machine learners can be designed to be more efficient and their limits can be easier understood. The thought framework presented is derived from the traditional experimental sciences which require published results to be self-contained with regards to reproducibility. In the practical exercises, we will work on calculating and measuring quantities like Memory Equivalent Capacity or generalization ratio for different machine learners and data sets and discuss how these quantities relate to reproducible experimental design.
\end{abstract}

\section{ACM Reference Format:}

Gerald Friedland. 2020. Reproducibility and Experimental Design for Machine Learning on Audio and Multimedia Data. In Proceedings of the 28th ACM International Conference on Multimedia (MM '20), October 12-16, 2020, Seattle, WA, USA. ACM, New York, NY, USA, 3 pages. https://doi.org/10. $1145 / 3394171.3418548$

\section{INTRODUCTION}

Everyday, thousands of videos, images, and websites appear on the Internet creating an ever-growing demand for methods to make them easier to retrieve, search, and index. YouTube alone claims that every minute, 100 hours worth of video material is uploaded. Most of these videos consist of consumer-produced, "unconstrained" videos from social media networks, such as YouTube uploads or Flickr content. Since many of these videos are reflecting people's everyday life experience, they constitute a corpus of never before seen scale for empirical research. But:

(1) What are the methods can cope with this amount of data?

(2) How does one approach the problems in a research setting, with or without thousands of compute cores at one's disposal?

(3) How does one budget and estimate the time needed to perform such experiments?

(4) What rigor has to be employed to make experiments robust in evaluations?

Permission to make digital or hard copies of part or all of this work for personal or classroom use is granted without fee provided that copies are not made or distributed for profit or commercial advantage and that copies bear this notice and the full citation on the first page. Copyrights for third-party components of this work must be honored.

For all other uses, contact the owner/author(s).

MM '20, October 12-16, 2020, Seattle, WA, USA

(C) 2020 Copyright held by the owner/author(s)

ACM ISBN 978-1-4503-7988-5/20/10.

https://doi.org/10.1145/3394171.3418548
Machine Learning in the Scientific Method

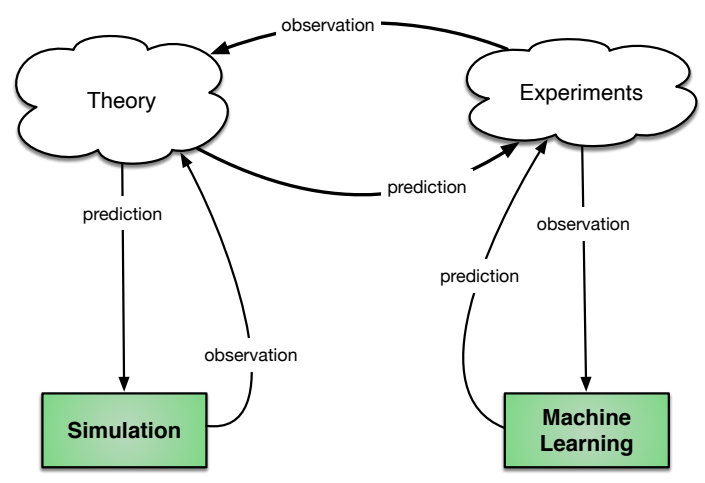

Figure 1: By understanding the position of machine learning in the scientific process and measuring the information flow in it, reproducibility becomes easier.

(5) What about reproducibility?

Here is a quick hint: The answer is not massive computational power.

There are many myth in Machine Learning that this tutorial will dispel. For example, speech recognition does not need the cloud and many machine learning tasks do not even need a GPU.

\section{OVERVIEW OF CONTENT}

This tutorial provides a theoretical and practical perspective on the experimental design for machine learning experiments on multimedia data.

(1) Scientific process revisited: information flow in Machine Learning (see also Figure 1)

(2) Mainstream practice: experimental setup with training and test set

(3) Experimental design principles understanding machine learning as compression

(4) The Occam's Razor, Memory Equivalent Capacity, and Generalization

(5) When to know that there is enough data: Capacity progression plots

(6) Hands on: Tensorflow Meter and capacity estimation tools

(7) Reproducibility: Learning from other disciplines

(8) The ACM standards of reproducibility

(9) Recommendations for the Multimedia community [4] 
Every field of engineering starts with measurements. Before we build a car, a plane, a bridge, or a computer chip, we measure something. It could be forces or impedances or tolerances or torque that we measure. But we measure first and then design around these measurements. Every engineer quotes some version of the trusted axiom, "Measure twice, cut once". Every field of science starts with measurements, too. Newton collected an enormous amount of data before coming up with the simple explanatory rule that describes and predicts the behavior of falling objects - the gravitational force equation. All scientific endeavors begin with observation, move on to hypothesizing and testing a rule, and then finally land on establishing a predictive model for some worldly phenomenon. Unfortunately, when working on supervised learning problems, modern data science and machine learning experts rely more on massive computation and guesswork (aka. "let's see if we can modify Alexnet for our specific problem") than on any kind of advance measurement. Of course, the results of guesses are anecdotes and which are mostly the opposite of reproducible. This tutorial presents a practical measurement framework for sizing machine learning models (neural networks, decision trees, etc.) and measuring the learnability of any labeled data set against these model types.

\section{MACHINE LEARNING MEASUREMENTS}

Consider the following thought experiment: How can one memorize these two sequences?

Sequence 1: "0, 20,40,60,80,100,120"

Sequence 2: "1,-4,1.1,52,2,9"

In the first case, the possibly most economical strategy to reproduce the numbers is to remember the initial number " 0 " and generalize the rule " +20 ". In the second case, it's not immediately clear what the underlying rule is so the quickest strategy to not lose any information is to memorize the entire sequence. These two examples illustrate how one can intuitively interpret memorization as worstcase generalization: Memorization only allows comparison against known input and requires verbatim storage while generalization is more effective (occupies less memory) than verbatim storage and at the same time allows to generate correct answers on similar, but unseen input (e.g., "200" would be the next number given the unseen input "180"), which is usually called "generalization". We can therefore define memorization as worst case generalization.

While the math behind generalization is generally unknown, the math behind memorization is well known and was established in 1949 by Claude Shannon. The unit of memorization is the binary digit (bit) and it is widely established that memory measurement does not differ based on the type of data one is memorizing (images, speech, text, etc...). The goal of machine learning is to create a model that generalizes. A model that only memorizes is generally said to "overfit the data". We also know that memorization can be more or less efficient. For example, one can store a 7-digit phone number on a hard disk with 1TB space. Similarly, machine learning models and the rules they contain can be even larger than needed for memorization of the input data. This may or may not be a problem. For example, the rule " +20 " could be represented as " $+1+1+1+1+1+1+1+1+1+1+1+1+1+1+1+1+1+1+1+1$ " and, while longer than the original input sequence, the rule still generalizes.
So while a machine learning model that implements a rule that has a longer description length than the input sequence can still generalize, practically speaking, we may not be able to distinguish easily between generalization and overfitting in such a case. However, when a rule implemented by a model has a shorter description length compared to its original training input and it achieves very high accuracy when tested, we can confidently say that the model generalizes. This is, to make our lives easier, we follow the principle of Occam's Razor [9] and define our goal in machine learning to be a maximal reduction of the number of parameters in the model while at the same time maximizing the accuracy. The connection between the number of parameters (seen as description length of the model) has been explained fundamentally by MacKay [7]. Given a machine learning model, the first question therefore is: How many parameters are minimally needed to encode and then decode the function represented by the training data? This is, what is the minimum number of parameters to reproduce the mapping represented by the training data with $100 \%$ accuracy. Which, as explained above, is equivalent to asking how many parameters would overfit the training data. In order to make this process measurable, we need to know how many bits of the training function each parameter can maximally memorize. This is dependent on the machine learner and also on the task that is being performed. For simplicity, in this tutorial, we will focus only on balanced binary (two-label) classification. For binary classification, the question of memory capacity was solved for decision trees by Shannon [8] and for neural networks by Friedland et al. [5]. Friedland et al. define what they call the Memory Equivalent Capacity. A machine learner's capacity is memory-equivalent to $\mathrm{N}$ bits when the machine learner is able to represent all $2^{N}$ binary labeling functions of $\mathrm{N}$ inputs. This means, that a machine learner is overfitting the training training data when its Memory Equivalent Capacity is greater or equal to the number of instances in the training data for a binary classification task. For example, when 10 training samples are given for a binary classification task and a perfect binary tree with depth 10 is used to classify the problem, the model overfits. Rules to derive the Memory Equivalent Capacity for neural networks by treating neurons like elements in an electrical circuit are derived in [3].

Using the explanation above, we are now able to measure generalization capability for balanced binary classification as the following ratio: $G=\frac{\text { \#correctly classified instances }}{\text { Memory Equivalent Capacity }}$, where $G$ is measured after the model is created and on all unique inputs the model can represent correctly. The larger $G$, the higher the generalization capability. As a consequence of the above derivation, if $G<=1$, the model is overfitting. Intuitively, we know the more samples of the training data that can be labeled correctly by each parameter of the model, the higher the probability that a parameter can correctly label an unseen input. Going back to the example from the beginning, the rule " +20 " is able to predict Sequence 1 with $100 \%$ accuracy but this model only takes 3 characters to memorize. This is, as explained above, the goal is to minimize Memory Equivalent Capacity while at the same time maximizing accuracy. Further explanation of the concept of Memory Equivalent Capacity and generalization are available as an online lecture [2] and, of course, will be part of the tutorial. 


\section{REFERENCES}

[1] A. Binet and T. Simon. Méthodes nouvelles pour le diagnostic du niveau intellectuel des anormaux. L'année Psychologique, 11(1):191-244, 1904.

[2] G. Friedland. Generalization. https://youtu.be/UZ5vhqDKyrY, 2019.

[3] G. Friedland. Reproducibility and Experimental Design for Machine Learning on Audio and Multimedia Data. In Proceedings of the 19th ACM International Conference on Multimedia, pages 2709--2710. ACM, October 2019.

[4] G. Friedland, R. Jia, J. Wang, B. Li, and N. Mundhenk. On the Impact of Perceptual Compression on Deep Learning. In Proceedings of the IEEE 3rd International Conference. on. Multimedia Information Processing and Retrieval. IEEE, August 2020.

[5] G. Friedland, A. Metere, and M. Krell. A Practical Approach to Sizing Neural Networks. arXiv preprint arXiv:1810.02328, October 2018 https://arxiv.org/abs/ 1810.02328 .

[6] L. Huang, A. D. Joseph, B. Nelson, B. I. Rubinstein, and J. Tygar. Adversarial machine learning. In Proceedings of the 4th ACM workshop on Security and Artificial Intelligence, pages 43-58. ACM, 2011.

[7] D. J. C. MacKay. Information Theory, Inference, and Learning Algorithms. Cambridge University Press, New York, NY, USA, 2003.

[8] C. E. Shannon. The Bell System Technical Journal. A mathematical theory of communication, 27:379-423, 1948.

[9] W. M. Thorburn. Occam's razor. 1915.

[10] Website. Tensorflow Meter. http://tfmeter.icsi.berkeley.edu, 2018. 